\title{
Andexanet Alfa: First Global Approval
}

\author{
Young-A Heo ${ }^{1}$ \\ Published online: 20 June 2018 \\ (c) Springer Nature 2018, corrected publication July/2018
}

\begin{abstract}
Intravenous andexanet alfa [coagulation factor Xa (recombinant), inactivated-zhzo; Andexxa ${ }^{\circledR}$ ] is a first-in-class recombinant modified factor Xa protein that has been developed by Portola Pharmaceuticals as a universal antidote to reverse anticoagulant effects of direct or indirect factor Xa inhibitors. In May 2018, andexanet alfa received its first global approval in the USA for use in patients treated with rivaroxaban and apixaban, when reversal of anticoagulant effects is required in life-threatening or uncontrolled bleeding. Intravenous andexanet alfa is under regulatory review in the EU and is undergoing clinical development in Japan. This article summarizes the milestones in the development of andexanet alfa leading to this first global approval for reversing anticoagulation of rivaroxaban and apixaban in adults.
\end{abstract}

\section{Introduction}

Direct [e.g. apixaban, edoxaban, rivaroxaban and betrixaban (which was approved in the USA in June 2017 [1])] or indirect (e.g. fondaparinux and the low-molecular-weightheparin enoxaparin) factor Xa inhibitors [2] are effective anticoagulants for the treatment and prevention of thromboembolism, and stroke prevention in atrial fibrillation [3]. Although these agents show a better bleeding risk profile compared with that of vitamin $\mathrm{K}$ antagonists (e.g. warfarin), the risk of bleeding complications still exists and the availability of a specific reversing agent would be beneficial $[4,5]$.

Intravenous andexanet alfa [coagulation factor $\mathrm{Xa}$ (recombinant), inactivated-zhzo; Andexxa $\left.{ }^{\circledR}\right]$ is a first-inclass recombinant modified factor Xa protein that has been developed by Portola Pharmaceuticals as a universal antidote to reverse the anticoagulant effects of direct or indirect factor Xa inhibitors [6]. Andexanet alfa acts as a decoy and binds to factor Xa inhibitors, neutralizing the anticoagulant effects of

The original version of this article was revised due to a retrospective Open Access request.

This profile has been extracted and modified from the AdisInsight database. AdisInsight tracks drug development worldwide through the entire development process, from discovery, through preclinical and clinical studies to market launch and beyond.

Young-A Heo

dru@adis.com

1 Springer, Private Bag 65901, Mairangi Bay, Auckland 0754, New Zealand factor Xa inhibitors by preventing the inhibitors from binding to endogenous factor $\mathrm{Xa}[7,8]$.

On 3 May 2018, andexanet alfa received US FDA accelerated approval for patients treated with rivaroxaban or apixaban who require reversal of the anticoagulant effects in life-threatening or uncontrolled bleeding [6]. The approval was based on the change in anti-factor Xa activity from baseline in two phase III studies in healthy volunteers (Sect. 2.3); continued approval for this indication is contingent upon the demonstration of improved haemostasis in patients in post-marketing studies [6,9]. Andexanet alfa is available as a $100 \mathrm{mg}$ vial for injection/infusion [9]. The recommended dosing regimen is a single intravenous bolus (400 or $800 \mathrm{mg}$ ) followed by a continuous infusion for up to $120 \mathrm{~min}$ ( 4 or $8 \mathrm{mg} / \mathrm{min}$ ); the dosage strengths chosen depends upon the last dose of rivaroxaban ( $\leq 10$ or $>10 \mathrm{mg}$ / unknown) or apixaban ( $\leq 5 \mathrm{or}>5 \mathrm{mg} /$ unknown), and time of the last dose of rivaroxaban or apixaban $(<8 \mathrm{~h} /$ unknown or $\geq 8$ h) [9]. The efficacy and safety of repeated administration of andexanet alfa has not been established. The US prescribing information carries a boxed warning regarding serious and life-threatening adverse events (AEs), including thromboembolic events, ischemic events, cardiac arrest and sudden deaths; symptoms and signs of these AEs should be monitored and treated appropriately [9].

Intravenous andexanet alfa is under regulatory review by the European Medicines Agency and is undergoing clinical development in Japan as a universal antidote for factor Xa inhibitors. 


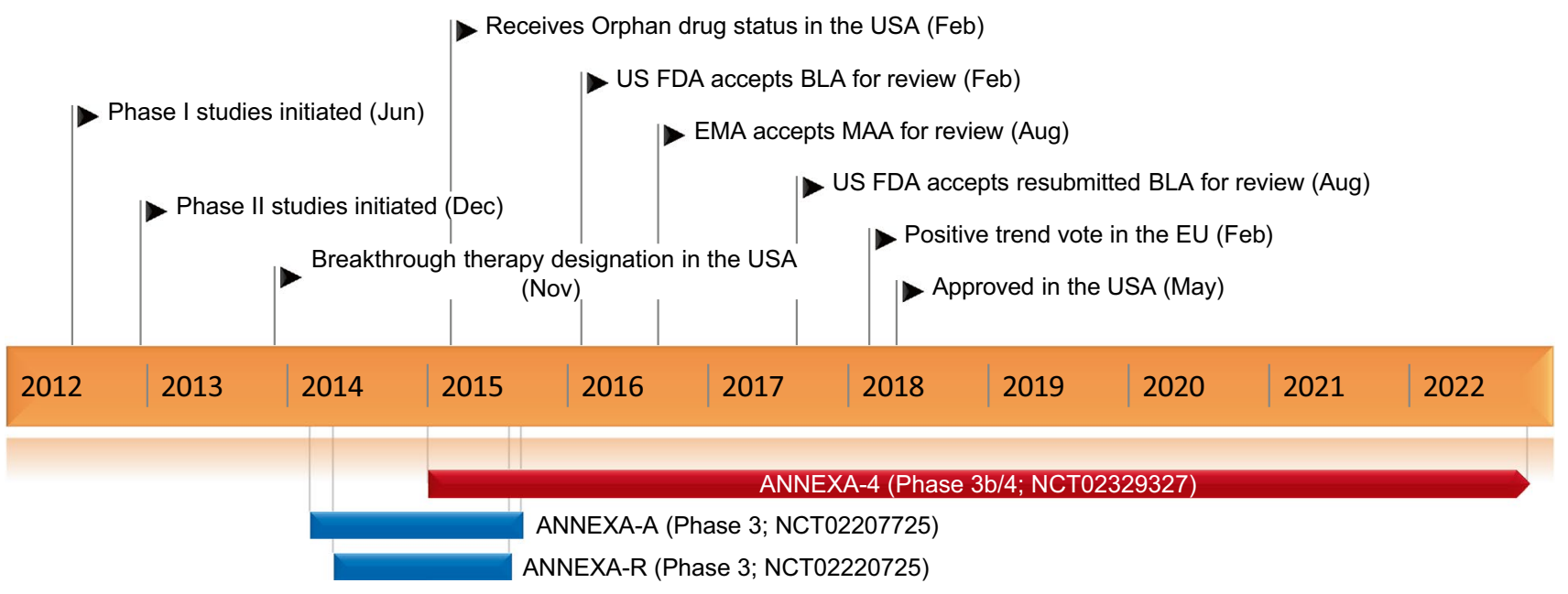

Key milestones in the development of andexanet alfa as a reversal agent for rivaroxaban and apixaban in adults who experience a major bleeding event. $B L A$ biologics license application, $M A A$ marketing authorization application

\subsection{Company Agreements and Patent Information}

In 2014, Portola Pharmaceuticals signed commercial supply agreements with CMC Biologics [10] and Lonza [11] for the global manufacturing of andexanet alfa.

Between 2012 and 2016, Portola Pharmaceuticals entered into ten separate non-exclusive clinical collaboration agreements with manufacturers of factor Xa inhibitors, including edoxaban (Daiichi Sankyo) [12-15], apixaban [Bristol-Myers Squibb (BMS) and Pfizer Inc.] [16-18] and rivaroxaban (Bayer Healthcare and Janssen) [19-21] to support phase II and III clinical trials in the USA and EU, and clinical development program in Japan to investigate the use of andexanet alfa as a universal antidote for factor $X$ a inhibitors. Pursuant to the terms of these agreements, Portola Pharmaceuticals received upfront payments and is eligible to receive additional milestone payments (related to development and regulatory approvals); these agreements are to remain in effect for the entire period of each trial.

In February 2016, BMS and Pfizer Inc. acquired licensing rights from Portola Pharmaceuticals for the development and commercialization of andexanet alfa in Japan [21]. Under this agreement, Portola Pharmaceuticals received an upfront payment and is eligible to receive regulatory and sales-based milestone payments, as well as double-digit royalties on net sales of andexanet alfa in Japan [21]. With the exception of Japan, Portola Pharmaceuticals retains the global right to commercialize andexanet alfa [21].

In February 2017, Portola Pharmaceuticals signed a royalty agreement with HealthCare Royalty Partners (HCR)
[22]. Under the terms of the agreement, Portola Pharmaceuticals received an upfront payment [22]. In May 2018, Portola Pharmaceuticals received a milestone payment following the approval of andexanet alfa in the USA [23]. In exchange, HCR is eligible to receive a tiered mid-singledigit royalty on any potential worldwide sales of andexanet alfa (total royalty payments are subject to a cap of $195 \%$ of the total payments funded by HCR) [23].

\subsection{Patent Information}

Portola Pharmaceuticals has patent protections for composition, manufacturing process and therapeutic use of andexanet alfa and its analogues in the USA until 2030. Andexanet alfa also has patent protection and pending patents in numerous countries including those of the EU, China, Singapore and New Zealand. Approved and pending international patents (if issued) will expire in 2028.

\section{Scientific Summary}

\subsection{Pharmacodynamics}

Andexanet alfa is a genetically modified variant of human factor Xa protein that is catalytically inactive but retains the structural similarity to that of endogenous factor Xa [2, 7-9]. The modifications mean that andexanet alfa is unable to cleave and activate prothrombin and cannot assemble into the prothrombinase complex [2, 7-9]. Andexanet alfa binds 
to apixaban, betrixaban, edoxaban and rivaroxaban with high affinity $(0.53-1.53 \mathrm{nmol} / \mathrm{L})$, similar to that seen with endogenous factor Xa [24, 25]. Consequently, andexanet alfa sequesters factor $\mathrm{Xa}$ inhibitors, leading to reversing the anticoagulant effects of factor Xa inhibitors and restoring the activity of endogenous factor Xa $[2,7,8]$.

In vitro, andexanet alfa dose-dependently reversed the anti-factor Xa activity of apixaban, betrixaban, edoxaban, rivaroxaban, enoxaparin and fondaparinux in human and rat plasma [24, 25]. In addition, andexanet alfa was shown to bind to tissue factor pathway inhibitor, an endogenous inhibitor of factor Xa, and transiently increased the level of prothrombin fragments 1 and 2, thrombin-antithrombin complex and D-dimer; this interaction can modulate tissuefactor initiated thrombin generation $[9,26]$.

In preclinical studies, andexanet alfa rapidly reversed the anticoagulant effects of factor Xa inhibitors (as measured by reduction in anti-factor Xa activity and unbound plasma concentrations of factor Xa inhibitors), thereby decreasing total blood loss in various animal models [24, $25,27-31]$. For instance, in a porcine model of polytrauma (a blunt liver injury and bilateral femur fractures) under apixaban anticoagulation, andexanet alfa significantly $(p<0.01)$ reduced total blood loss compared with vehicle when andexanet alfa was administered as a bolus (1264 vs. $3903 \mathrm{~mL}$ ) or a bolus plus $2 \mathrm{~h}$ infusion (1202 vs. $3903 \mathrm{~mL}$ ); survival was $100 \%$ in the andexanet alfa group. Furthermore, anti-factor Xa levels of apixaban were close to zero with andexanet alfa and peak thrombin generation was normalized [28].
In a series of dose-ranging, proof-of-concept phase II trials, andexanet alfa demonstrated dose-dependent reversal of anticoagulation of direct or indirect factor Xa inhibitors (i.e. apixaban, rivaroxaban, betrixaban, edoxaban and enoxaparin) in healthy volunteers [32-35]. Following dosing with one of the factor $\mathrm{Xa}$ inhibitors, volunteers received andexanet alfa or placebo. Within $\approx 2 \mathrm{~min}$ after administration, andexanet alfa rapidly reversed the anticoagulant effects of factor Xa inhibitors, as shown by the reduction in anti-factor Xa levels and restoration of normal thrombin generation and clotting time [32-35]. The pharmacodynamic half-life of andexanet alfa is $\approx 1 \mathrm{~h} \mathrm{[36].}$ The reduction in anti-factor Xa levels was sustained during andexanet alfa treatment and the levels returned to placebo levels $\approx 2 \mathrm{~h}$ after the bolus or after the end of infusion [32-35], which was consistent with the short pharmacodynamic half-life of andexanet alfa [36].

\subsection{Pharmacokinetics}

Intravenous andexanet alfa exposure increased in a dose-proportional manner at bolus doses $>30 \mathrm{mg}$ in healthy subjects [35]. The mean apparent volume of distribution of andexanet alfa was $\approx 5 \mathrm{~L}$ [9]. Andexanet alfa was rapidly eliminated (clearance $\approx 4.3 \mathrm{~L} / \mathrm{h}$ ) and the terminal elimination half-life ranged from 5 to $7 \mathrm{~h}$. The pharmacokinetics of andexanet alfa were not affected by age and by the presence of oral apixaban (5 mg twice daily for 6 days) or oral rivaroxaban (20 mg once daily for 6 days) [9].

\section{Features and properties of andexanet alfa [coagulation factor Xa (recombinant), inactivated-zhzo]}

\begin{tabular}{|c|c|}
\hline Alternative names & $\begin{array}{l}\text { AndexXa; AnXa; IndexXa; PRT-064445; PRT-4445; PRT-44545; recombinant factor-Xa-Portola; } \\
\text { rfXa-inhibitor-antidote }\end{array}$ \\
\hline Class & Antidotes; antihaemorrhagics; disulfides; recombinant proteins \\
\hline Mechanism of action & Modified factor Xa recombinant protein; blood coagulation stimulants \\
\hline Route of administration & Intravenous \\
\hline Pharmacodynamics & Binds to factor Xa inhibitors with high affinity and reverses the anticoagulant activity \\
\hline Pharmacokinetics & $\begin{array}{l}\text { Dose-proportional pharmacokinetics; mean apparent volume of distribution } \approx 5 \mathrm{~L} \text {; mean elimination } \\
\text { half-life } 5-7 \mathrm{~h}\end{array}$ \\
\hline Most frequent adverse events & Urinary tract infections, pneumonia \\
\hline \multicolumn{2}{|l|}{ ATC codes } \\
\hline WHO ATC code & B02 (antihemorrhagics) \\
\hline EphMRA ATC code & B2B (antagonists (antidotes to anticoagulants)) \\
\hline Chemical name & $\begin{array}{l}\text { des-(6-39)-Human blood-coagulation factor X light chain (98-108')-disulfide with [185'-alanine } \\
(\mathrm{S}>\mathrm{A}) \text { ] human activated factor Xa heavy chain }\end{array}$ \\
\hline
\end{tabular}




\subsection{Therapeutic Trials}

In two randomized, double-blind, placebo-controlled phase III trials, intravenous andexanet alfa reversed the anticoagulant effects of apixaban (ANNEXA-A; NCT02207725) and rivaroxaban (ANNEXA-R; NCT02220725) in healthy older volunteers (aged 50-75 years) [36]. Before participants were randomized to receive andexanet alfa or placebo on day 4 , those enrolled in ANNEXA-A $(n=64)$ received oral apixaban $5 \mathrm{mg}$ twice daily for 3.5 days and those in ANNEXA-R $(n=80)$ received oral rivaroxaban $20 \mathrm{mg}$ once daily for 4 days so that steady-state plasma levels of the anticoagulant agents was achieved. In ANNEXA-A, andexanet alfa recipients received an intravenous bolus of $400 \mathrm{mg}$ (part 1) or an intravenous bolus of $400 \mathrm{mg}$ followed by a continuous infusion of $4 \mathrm{mg} / \mathrm{min}$ for $120 \mathrm{~min}$ (part 2). In ANNEXA-R, andexanet alfa recipients received an intravenous bolus of $800 \mathrm{mg}$ (part 1) or an intravenous bolus of $800 \mathrm{mg}$ followed by a continuous infusion of $8 \mathrm{mg} / \mathrm{min}$ for $120 \mathrm{~min}$ (part 2) [36].

Andexanet alfa significantly $(p<0.001)$ reduced antifactor Xa activity from baseline to nadir (primary endpoint) compared with placebo in both part 1 and 2 of ANNEXA-A (part 1: 94 vs. $21 \%$; part 2: 92 vs. $33 \%$ ) and ANNEXA-R (part 1: 92 vs. 18\%; part 2: 97 vs. 45\%) [36]. Andexanet alfa rapidly reduced anti-factor $\mathrm{Xa}$ activity of apixaban and rivaroxaban within 2-5 min of administration and the effect was sustained during andexanet alfa treatment. The anti-factor $\mathrm{Xa}$ levels returned to placebo levels over $1-2 \mathrm{~h}$ after the bolus (part 1) or after the end of the infusion (part 2). $\geq 80 \%$ of anti-factor Xa activity was reversed by all andexanet alfa recipients compared with no placebo recipients $(p<0.001)$. Andexanet alfa also significantly $(p<0.001)$ reduced unbound concentrations of apixaban and rivaroxaban compared with placebo; unbound concentrations of apixaban and rivaroxaban returned to placebo level within $1-3 \mathrm{~h}$ after the bolus or the infusion. Moreover, a significantly $(p<0.001)$ higher proportion of andexanet alfa than placebo recipients restored thrombin generation in ANNEXA-A (part 1: 100 vs. $11 \%$; part 2: 100 vs. $25 \%$ ) and ANNEXA-R (part 1: 96 vs. $7 \%$; part 2: 100 vs. $0 \%$ ) within $2-5$ min after treatment. Prothrombin fragments 1 and 2, thrombin-antithrombin complex and D-dimer were transiently elevated in andexanet alfa recipients; the levels returned to the normal range within 24-72 h [36].
In the ongoing, multinational, single-arm, open-label phase IIIb/IV ANNEXA-4 study (NCT02329327), three interim analyses (after 67 [37], 185 [9] and 227 [38] patients were treated) showed that andexanet alfa reduced anti-factor Xa activity in patients who presented with acute major bleeding after taking factor Xa inhibitors $[9,37,38]$. ANNEXA-4 included patients (aged $\geq 18$ years) with major bleeding who received the last dose of apixaban, rivaroxaban, edoxaban or enoxaparin $\leq 18 \mathrm{~h}$ prior to administration of andexanet alfa [37]. Patients received intravenous andexanet alfa bolus ( $400 \mathrm{or} 800 \mathrm{mg}$ ) followed by a 120-min infusion (480 or $960 \mathrm{mg}$ ); the dosage depended on the type of factor Xa inhibitor and the time since the last dose [37]. During the trial, there was a protocol amendment to change the time since the last dose of factor Xa inhibitor for dosing andexanet alfa from $>7$ and $\leq 7 \mathrm{~h} /$ unknown to $\geq 8$ and $<8 \mathrm{~h} /$ unknown (data on file). A pharmacokinetic/pharmacodynamics model of andexanet alfa based on data from healthy volunteers supported the selected andexanet alfa dosing regimen for this population [39].

In the most recent interim analysis of ANNEXA-4 (cut-off date of 20 October 2017; $n=227$ ), the mean patient age was 77 years; $78 \%$ of patients received anticoagulation treatment for atrial fibrillation, $61 \%$ had intracranial bleeding, $27 \%$ had gastrointestinal bleeding, and the mean time from presentation to the initiation of the andexanet alfa treatment was $4.7 \mathrm{~h} \mathrm{[38].} \mathrm{According} \mathrm{to} \mathrm{a} \mathrm{descriptive} \mathrm{preliminary} \mathrm{analysis,}$ by the end of infusion, andexanet-alfa reduced anti-factor Xa activity from baseline to nadir by 91,87 and $73 \%$ in patients who were receiving apixaban $(n=105)$, rivaroxaban $(n=75)$ or enoxaparin ( $n=16)$, respectively (co-primary endpoint). At $12 \mathrm{~h}$ after the end of the infusion, the reduction of antifactor Xa activity in the respective groups was decreased to 35,60 and $52 \%$. Of the 132 evaluable patients, $83 \%$ achieved excellent or good haemostasis $12 \mathrm{~h}$ after the andexanet alfa infusion (co-primary endpoint). The effective haemostasis provided by andexanet alfa was consistently seen regardless of type of anticoagulant treatment (apixaban, rivaroxaban or enoxaparin), sex, site of bleeding (gastrointestinal, intracranial or other), age (aged $<65,65-75$, or $>75$ years) or the dose of andexanet alfa (low or high) [38].

Further supportive evidence for the efficacy of andexanet alfa in reducing anti-factor Xa activity of direct or indirect factor Xa inhibitors is available from several dose-ranging, proof-of-concept phase II studies in healthy volunteers (Sect. 2.1) [32-35]. 
Key clinical trials of andexanet alfa, sponsored by Portola Pharmaceuticals

\begin{tabular}{|c|c|c|c|c|c|}
\hline $\operatorname{Drug}(\mathrm{s})$ & Indication & Phase & Status & Location(s) & Identifier \\
\hline $\begin{array}{l}\text { Andexanet alfa, } \\
\text { placebo }\end{array}$ & $\begin{array}{l}\text { Reversal of anticoagulant activity of apixaban in } \\
\text { healthy volunteers }\end{array}$ & III & Completed & USA & NCT02207725 (ANNEXA-A) \\
\hline $\begin{array}{l}\text { Andexanet alfa, } \\
\text { placebo }\end{array}$ & $\begin{array}{l}\text { Reversal of anticoagulant activity of rivaroxaban in } \\
\text { healthy volunteers }\end{array}$ & III & Completed & USA & NCT02220725 (ANNEXA-R) \\
\hline Andexanet alfa & $\begin{array}{l}\text { Reversal of anticoagulant activity of several factor } \\
\text { Xa inhibitors in patients with acute major bleeding }\end{array}$ & $\mathrm{IIIb} / \mathrm{IV}$ & Ongoing & Multinational & $\begin{array}{l}\text { NCT02329327; EudraCT2015- } \\
\text { 001785-26 (ANNEXA-4) }\end{array}$ \\
\hline $\begin{array}{l}\text { Andexanet alfa, } \\
\text { placebo }\end{array}$ & $\begin{array}{l}\text { Reversal of anticoagulant activity of several factor } \\
\text { Xa inhibitors in healthy volunteers }\end{array}$ & II & Completed & USA & NCT01758432 \\
\hline $\begin{array}{l}\text { Andexanet alfa, } \\
\text { placebo }\end{array}$ & $\begin{array}{l}\text { Reversal of anticoagulant activity of betrixaban in } \\
\text { healthy volunteers }\end{array}$ & II & Ongoing & USA & NCT03330457 \\
\hline $\begin{array}{l}\text { Andexanet alfa, } \\
\text { placebo }\end{array}$ & $\begin{array}{l}\text { Reversal of anticoagulant activity of several factor } \\
\text { Xa inhibitors in healthy Japanese and Caucasian } \\
\text { volunteers }\end{array}$ & II & Ongoing & USA & NCT03310021 \\
\hline $\begin{array}{l}\text { Generation } 1 \text { and } \\
\text { generation } 2 \\
\text { andexanet alfa, } \\
\text { placebo }\end{array}$ & $\begin{array}{l}\text { Reversal of anticoagulant activity of several factor } \\
\text { Xa inhibitors in healthy volunteers }\end{array}$ & I & Ongoing & USA & NCT03083704 \\
\hline
\end{tabular}

\subsection{Adverse Events}

Intravenous andexanet alfa was generally well tolerated in healthy volunteers [36] and the most common AE occurring in $\geq 3 \%$ of volunteers receiving andexanet alfa was infusionrelated reactions [9]. The tolerability profile of andexanet alfa in patients with acute major bleeding who received factor Xa inhibitors was generally similar to that reported of other approved reversal agents [38] and the most common AEs occurring in $\geq 5 \%$ of patients receiving andexanet alfa were urinary tract infections and pneumonia [9].

In an interim safety analysis of 227 patients in the ongoing ANNEXA-4 trial, patients were followed for 30 days after andexanet alfa treatment [38]. In the first 30 days after treatment, thrombotic events (stroke, deep-vein thrombosis and heart attack) occurred in 24 patients (11\%); only nine patients restarted anticoagulation therapy before the time of thrombotic event [38]. Anticoagulant therapy should be restarted as soon as medically appropriate as anticoagulation-treated patients are predisposed to thrombotic events due to their underlying medical condition [9, 38]. A total of 27 patients $(12 \%)$ had died, of which 11 deaths were due to cardiovascular reasons [38].

In the pooled analysis of clinical trials in healthy volunteers, similar proportions of andexanet alfa $(n=223)$ and placebo $(n=94)$ recipients reported AEs (54 vs. $57 \%)$, none of which were serious or severe [9]. The only AE occurring with a higher incidence in andexanet alfa than placebo recipients was infusion-related reactions (18 vs. 6\%), which were of mild to moderate intensity and were generally manageable without treatment (90\%) [9].

As with all therapeutic proteins, there is a potential for immunogenicity with andexanet alfa [9]. Among the 145 healthy subjects treated with andexanet alfa, 17\% had low titers of anti-andexanet alfa antibodies. The pattern of antibody response in bleeding patients has been similar to that seen in healthy volunteers. In ANNEXA-4, $6 \%$ of 98 patients developed antibodies to andexanet alfa within 30 days of treatment but none developed neutralizing antibodies. To date, antibodies with cross-reactivity to factor X or Xa were neither detected in healthy subjects, nor in bleeding patients [9].

\subsection{Ongoing Clinical Trials}

There are four ongoing clinical trials of andexanet alfa. The multinational, open-label phase IIIb/IV ANNEXA-4 (NCT02329327) is still underway. A randomized, doubleblind and placebo-controlled phase II trial (NCT03310021) to evaluate the efficacy of andexanet alfa for reversing anticoagulation of apixaban, rivaroxaban and edoxaban in healthy Japanese and Caucasian volunteers has been initiated. A randomized, double-blind and placebo-controlled phase II trial (NCT03330457) is currently ongoing to evaluate the efficacy of andexanet alfa for reversing anticoagulation of betrixaban in healthy volunteers. Lastly, a randomized, double-blind and placebo-controlled phase I trial (NCT03083704) is currently ongoing to evaluate pharmacokinetics, pharmacodynamics, safety and tolerability of second generation andexanet alfa in healthy volunteers.

\section{Current Status}

Andexanet alfa received its first global approval on 3 May 2018 in the USA for use in adults treated with rivaroxaban and apixaban, when reversal of its anticoagulant effects 
is required because of life-threatening or uncontrolled bleeding.

\section{Compliance with Ethical Standards}

Funding The preparation of this review was not supported by any external funding.

Conflict of interest During the peer review process the manufacturer of the agent under review was offered an opportunity to comment on the article. Changes resulting from any comments received were made by the author on the basis of scientific completeness and accuracy. Young-A Heo is a salaried employee of Adis/Springer, is responsible for the article content and declares no relevant conflicts of interest.

Open Access This article is distributed under the terms of the Creative Commons Attribution-NonCommercial 4.0 International License (http://creativecommons.org/licenses/by-nc/4.0/), which permits any noncommercial use, duplication, adaptation, distribution and reproduction in any medium or format, as long as you give appropriate credit to the original author(s) and the source, provide a link to the Creative Commons license and indicate if changes were made.

\section{References}

1. US FDA. FDA approved betrixaban (Bevyxxa, Portola) for the prophylaxis of venous thromboembolism (VTE) in adult patients [media release]. 23 June 2017. http://www.fda.gov.

2. Kaatz S, Bhansali H, Gibbs J, et al. Reversing factor Xa inhibitors-clinical utility of andexanet alfa. J Blood Med. 2017;8:141-9.

3. Levy JH, Douketis J, Weitz JI. Reversal agents for nonvitamin $\mathrm{K}$ antagonist oral anticoagulants. Nat Rev Cardiol. 2018;15(5):273-81.

4. Ansell JE. Reversing the effect of oral anticoagulant drugs: established and newer options. Am J Cardiovasc Drugs. 2016;16(3):163-70.

5. Hu TY, Vaidya VR, Asirvatham SJ. Reversing anticoagulant effects of novel oral anticoagulants: role of ciraparantag, andexanet alfa, and idarucizumab. Vasc Health Risk Manag. 2016;12:35-44.

6. Portola Pharmaceuticals. U.S. FDA approves Portola Pharmaceuticals Andexxa ${ }^{\circledR}$, the first and only antidote for the reversal of factor Xa inhibitors [media release]. 3 May 2018

7. Goldin M, Hughes GJ, Choudhary Z, et al. Reversal of anticoagulation: therapeutic advances and clinical guidelines. Am J Ther. 2018;25(1):e44-52.

8. Lippi G, Sanchis-Gomar F, Favaloro EJ. Andexanet: effectively reversing anticoagulation. Trends Pharmacol Sci. 2016;37(6):613-4.

9. Portola Pharmaceuticals. Andexxa (coagulation factor Xa (recombinant), inactivated-zhzo): US prescribing information. 2018. http://www.fda.gov/. Accessed 15 May 2018.

10. CMC Biologics. CMC Biologics enters into commercial supply agreement with Portola Pharmaceuticals for manufacturing of first-in-class factor Xa inhibitor antidote andexanet alfa [media release]. 2 July 2014.

11. Portola Pharmaceuticals. Portola Pharmaceuticals announces commercial supply agreement for andexanet alfa with Lonza [media release]. 16 Oct 2014

12. Portola Pharmaceuticals. Portola to initiate phase 2 study evaluating the safety and effectiveness of its factor Xa inhibitor antidote
PRT4445 in reversing the anticoagulant activity of investigational factor Xa inhibitor edoxaban [media release]. 26 June 2013.

13. Portola Pharmaceuticals. Portola Pharmaceuticals enters into clinical collaboration agreement with Daiichi Sankyo for phase 3 studies of factor Xa inhibitor antidote, andexanet alfa, and edoxaban [media release]. 7 July 2014.

14. Portola Pharmaceuticals. Portola Pharmaceuticals enters into clinical collaboration agreement with Daiichi Sankyo to develop andexanet alfa with edoxaban in Japan [media release]. 4 Apr 2016.

15. Portola Pharmaceuticals. Portola Pharmaceuticals expands clinical collaboration agreement with Daiichi Sankyo worth up to \$25 million to develop Andexxa ${ }^{\mathrm{TM}}$ (andexanet alfa) in Germany [media release]. 1 Nov 2016.

16. Portola Pharmaceuticals. Portola, Bristol-Myers Squibb and Pfizer sign clinical collaboration agreement to study Eliquis and Portolas universal factor Xa inhibitor antidote PRT4445 [media release]. 1 Nov 2012.

17. Portola Pharmaceuticals. Portola Pharmaceuticals enters second clinical collaboration agreement with Bristol-Myers Squibb and Pfizer to study andexanet alfa* (PRT4445), investigational factor $\mathrm{Xa}$ inhibitor reversal agent, with Eliquis [media release]. 13 Jan 2014.

18. Bristol-Myers Squibb, Pfizer. Bristol-Myers Squibb and Pfizer sign collaboration with Portola Pharmaceuticals to develop and commercialize investigational andexanet alfa in Japan [media release]. 1 Feb 2016.

19. Portola Pharmaceuticals. Portola enters into clinical collaboration agreement with Bayer and Janssen for phase 2 study of universal factor Xa inhibitor antidote, PRT4445, and Xarelto ${ }^{\circledR}{ }^{\text {[media }}$ release]. 5 Feb 2013.

20. Portola Pharmaceuticals. Portola Pharmaceuticals enters into clinical collaboration agreement with Bayer and Janssen for phase 3 studies of andexanet alfa*, investigational factor Xa inhibitor reversal agent, and Xarelto ${ }^{\circledR}$ [media release]. 3 Feb 2014.

21. Portola Pharmaceuticals. Portola Pharmaceuticals enters into licensing agreements for investigational agent andexanet alfa in Japan worth up to \$120 million [media release]. 1 Feb 2016.

22. Portola Pharmaceuticals. Portola Pharmaceuticals signs $\$ 150$ million royalty agreement with HealthCare Royalty Partners for development and commercialization of andexanet alfa [media release]. 3 Feb 2017.

23. Portola Pharmaceuticals. Portola Pharmaceuticals receives $\$ 100$ million milestone payment from HealthCare Royalty Partners for FDA approval of Andexxa ${ }^{\circledR}$ [media release]. 24 May 2018.

24. Lu G, DeGuzman FR, Hollenbach SJ, et al. A specific antidote for reversal of anticoagulation by direct and indirect inhibitors of coagulation factor Xa. Nat Med. 2013;19(4):446-51.

25. Lu G, Pine P, Leeds JM, et al. Andexanet alfa effectively reverses edoxaban anticoagulation effects and associated bleeding in a rabbit acute hemorrhage model. PLoS One. 2018;13(3):e0195122.

26. Lu G, Lin JP, Curnutte JT, et al. Effect of andexanet-TFPI interaction on in vitro thrombin formation and coagulation markers in the TF-pathway [abstract]. In: Blood conference: 59th annual meeting of the American Society of Hematology, ASH. 2017.

27. Milling TJ Jr, Kaatz S. Preclinical and clinical data for factor Xa and "universal" reversal agents. Am J Emerg Med. 2016;34(11s):39-45.

28. Grottke O, Akman N, Conley PB, et al. The impact of andexanet alfa in a porcine polytrauma model under apixaban anticoagulation: investigation of hemostatic safety and efficacy [abstract no. 20205]. In: Circulation Conference: Resuscitation Science Symposium, ReSS, vol 136, Suppl 1. 2017.

29. Conley PB, Grottke O, Braunschweig T, et al. Prevention of exanguination under apixaban anticoagulation using andexanet 
alfa in a polytrauma model [abstract no. 177]. Neurocrit Care. 2017;27(Suppl. 2):S198.

30. Conely PB, Pine P, Deguzman F, et al. Andexanet alfa reduces betrixaban-induced blood loss in a rabbit liver laceration model of acute bleeding [abstract no. P6208]. Eur Heart J. 2017;38(Suppl 1):ehx493.

31. Lu G, Pine P, deGuzman F, et al. Reversal of anticoagulation effects of rivaroxaban and associated bleeding in a rabbit acute hemorrhage model by andexanet alfa vs. coagulation replacement factors. Neurology. 2017;88(16 Suppl) [abstract no. P5.054].

32. Crowther M, Lu G, Leeds J, et al. Andexanet alfa reverses anticoagulation induced by betrixaban in healthy volunteers [abstract no. 202]. Crit Care Med. 2018;46(1 Suppl):83.

33. Crowther M, Lu G, Conley P, et al. Reversal of factor Xa inhibitors-induced anticoagulation in healthy subjects by andexanet alfa [abstract no. 455]. Crit Care Med. 2014;42(12 Suppl):A1469.

34. Crowther M, Levy GG, Lu G, et al. A phase 2 randomized, double-blind, placebo-controlled trial demonstrating reversal of edoxaban-induced anticoagulation in healthy subjects by andexanet alfa (PRT064445), a universal antidote for factor Xa (FXa) inhibitors [abstract]. In: 56th annual meeting of the American Society of Hematology, ASH. 2014.

35. Siegal D, Lu G, Leeds JM, et al. Safety, pharmacokinetics, and reversal of apixaban anticoagulation with andexanet alfa. Blood Adv. 2017;1(21):1827-38.

36. Siegal DM, Curnutte JT, Connolly SJ, et al. Andexanet alfa for the reversal of factor Xa inhibitor activity. N Engl J Med. 2015;373(25):2413-24.

37. Connolly SJ, Milling TJ Jr, Eikelboom JW, et al. Andexanet alfa for acute major bleeding associated with factor Xa inhibitors. N Engl J Med. 2016;375(12):1131-41.

38. Connolly S, Milling T, Eikelboom JW, et al. Andexanet alfa in factor Xa inhibitor-associated acute major bleeding [abstract plus slide presentation]. In: American College of Cardiology's 67th annual scientific session \& expo (ACC18). 2018.

39. Leeds J, Mandema J, Lu G, et al. Pharmacokinetic/pharmacodynamic modeling of andexanet alfa dose in acute major bleeding [abstract no. 33]. Crit Care Med. 2018;46(1 Suppl.):17. 